\title{
Merlina y nuevos tratamientos de schwannomas vestibulares en pacientes con neurofibromatosis tipo 2
}

\section{Merlin and new treatments for vestibular schwannomas in patients with Neurofibromatosis type 2}

\author{
Felipe Cardemil', Paul H. Délano ${ }^{2,3}$.
}

\begin{abstract}
RESUMEN
Los schwannomas vestibulares son tumores benignos que habitualmente se presentan en forma esporádica y unilateral, pero pueden aparecer de manera bilateral en el contexto de una neurofibromatosis tipo 2 (NF2). En aquellos asociados a NF2 se han identificado mutaciones del gen NF2 que codifica para merlina, una proteína citoplasmática que se localiza primariamente en protrusiones celulares ricas en actina, y en sitios de contacto entre células y matriz extracelular. La evidencia sugiere que merlina ejerce un rol como proteína supresora de tumores ya que regula la cascada de activación de diversos tipos de receptores de factores de crecimiento celular. De esta manera, el déficit de merlina provoca un patrón de proliferación celular aumentado, alteraciones del citoesqueleto, apoptosis disminuida, y un incremento de la adhesión a la matriz extracelular. Se han desarrollado terapias clínicas para la NF2 con anticuerpos monoclonales e inhibidores dirigidos contra distintas moléculas involucradas en las cascadas de señalización celular moduladas por merlina. En este artículo se revisan y discuten los mecanismos celulares dependientes de merlina y los diversos estudios clínicos y experimentales que se han probado en pacientes con NF2.
\end{abstract}

Palabras claves: Merlina, neurofibromatosis tipo 2, schwannoma vestibular, anticuerpos monoclonales, bevacizumab.

\begin{abstract}
Vestibular schwannomas are benign tumors that may occur bilaterally in the context of neurofibromatosis type 2 (NF2). A mutation in the NF2 gene coding for merlin protein has been identified in those cases associated with NF2. Merlin is a cytoplasmic protein localized in actin rich cell protrusions, and near contact sites between cells and extracellular matrix. The evidence suggests that merlin plays a role as tumor suppressor protein, regulating the activation cascade of different types of receptors for cell growth factors. Thus, merlin deficiency causes a pattern of increased cell proliferation, cytoskeletal alterations, decreased apoptosis and increased cell adhesion to the extracellular matrix.
\end{abstract}

\footnotetext{
Servicio de Otorrinolaringología, Hospital Barros Luco Trudeau, Universidad de Chile.

Servicio de Otorrinolaringología, Hospital Clínico de la Universidad de Chile.

Programa de Fisiología y Biofísica, ICBM, Facultad de Medicina, Universidad de Chile.
} 
Several clinical therapies have been developed for NF2 patients including monoclonal antibodies and inhibitors directed against different molecules involved in cell signaling cascades modulated by merlin. In this article we review and discuss cellular mechanisms dependent of merlin and some clinical and experimental studies that have been studied in patients with NF2.

Key words: Merlin, Neurofibromatosis type 2, Vestibular Schwannoma, monoclonal antibodies, Bevacizumab

\section{INTRODUCCIÓN}

Los schwannomas son tumores encapsulados derivados de las células de Schwann, que no invaden a los axones neurales, y que habitualmente comprometen al nervio vestibular. La neurofibromatosis tipo 2 (NF2) es una enfermedad de herencia autosómica dominante que se caracteriza por la presencia de schwannomas vestibulares (SchV) bilaterales, meningiomas y en ocasiones ependimomas (Tabla 1), causada por una mutación del gen NF2 del cromosoma $22^{1}$. Otros nombres por los que se ha conocido a esta condición incluyen neurofibromatosis central, neurofibromatosis acústica bilateral, neurofibromatosis craneal, schwannomatosis central, neurofibromatosis universal, síndrome de neuroma acústico bilateral familiar,

Tabla 1. Criterios clínicos de neurofibromatosis tipo 2

\begin{tabular}{|ll}
\hline Criterios de Manchester \\
\hline A & Schwannomas vestibulares bilaterales. \\
B & $\begin{array}{l}\text { Familiar de primer grado con NF2 y schwannoma vestibular unilateral o } 2 \text { de los siguientes: } \\
\text { meningioma, schwannoma, glioma, neurofibroma, opacidad lenticular subcapsular posterior. }\end{array}$ \\
D & $\begin{array}{l}\text { Schwannoma vestibular unilateral y } 2 \text { de los siguientes: meningioma, schwannoma, glioma, } \\
\text { neurofibroma, opacidad lenticular subcapsular posterior. }\end{array}$ \\
C & Meningiomas múltiples (2 o más) y schwannoma vestibular unilateral o 2 de los siguientes: \\
Schwannoma, glioma, neurofibroma, catarata.
\end{tabular}

NNFF: National Neurofibromatosis Foundation.

(Tabla modificada de Hanemann. Brain 2008; 131: 606-615). 
neurinomatosis, y otros. También se le conoce con el epónimo de "síndrome de Wishart-Gardner-Eldridge"2. Se ha confundido históricamente con el síndrome de Von Recklinghausen, pero desde 1987 un grupo de consenso del National Institute of Health diferenció las manifestaciones clínicas vinculadas con el síndrome de Von Recklinghausen (neurofibromatosis periférica), que pasó a ser llamada neurofibromatosis tipo 1 (NF1), de las vinculadas con el síndrome de Wishart-GardnerEldridge (neurofibromatosis central), que pasó a denominarse $\mathrm{NF}^{3}$. Una tercera enfermedad que suele confundirse es la denominada schwannomatosis ${ }^{4}$, la que es una condición usualmente esporádica (a pesar que existen reportes de familias que presentaban transmisión autosómica dominante ${ }^{5}$ ), no acorta la expectativa de vida (pero sí la calidad de vida debido a dolores asociados a los tumores), y presenta múltiples schwannomas, pero no SchV bilaterales. Ocasionalmente desarrollan meningiomas, pero no desarrollan ependimomas ni alteraciones oculares. Se caracteriza por el desarrollo de múltiples schwannomas en nervios periféricos y raíces espinales, con ausencia de placas intracutáneas ${ }^{1}$.

La primera descripción de la evolución clínica y datos posmortem de la NF2 data de hace casi dos siglos, y correspondió a un paciente que presentaba hipoacusia bilateral, cefalea intratable y vómitos, falleciendo a los 21 años de edad². La NF2 es mucho más infrecuente que la NF1, presentando una incidencia de entre $1: 25.000$ a 1:50.000 1,3 . Se describe una herencia autosómica dominante y la penetrancia genética va más allá de 95\%. La NF2 se manifiesta con más frecuencia en el segundo y tercer decenio de la vida, siendo muy inusual que ocurra después de los 60 años de edad. Los síntomas se atribuyen a SchV, meningiomas craneales, y tumores espinales. La presentación de la NF2 puede variar de manera considerable, pero se ha dividido en 2 subtipos basados en la gravedad de la enfermedad; el subtipo Wishart es el más grave, y se caracteriza por un inicio temprano de los tumores, evolución más rápida de la enfermedad, y presencia de otros tumores múltiples además de los SchV. Por otra parte, el subtipo Gardner es más leve, se identifica por un comienzo tardío de los síntomas, evolución más benigna, y presencia casi exclusiva de SchV. A pesar de esto, muchos pacientes con NF2 presentan formas intermedias de la enfermedad².

Los schwannomas pueden ocurrir: (i) como parte de la NF2, (ii) de manera esporádica, 0 (iii) como parte de la schwannomatosis. Son tumores benignos y es extremadamente poco frecuente que un schwannoma se convierta en maligno. Corresponden a los tumores más frecuentes que afectan a los nervios periféricos, ubicándose el $50 \%$ en cabeza y cuello. Se ha descrito que hasta en $3 \%$ a $4 \%$ de las autopsias se encuentran schwannomas de nervios craneales ${ }^{6}$. El par craneal más frecuentemente afectado es la rama vestibular del VIII par (Io que corresponde a un SchV), seguido por el V par, mientras que los que afectan el nervio vago 0 facial son aún menos frecuentes ${ }^{7,8}$. Los SchV constituyen alrededor del $8 \%$ de los tumores intracraneales, comprendiendo cerca del $80 \%$ de los tumores ubicados en el ángulo pontocerebeloso ${ }^{1,9}$. La mayoría de los casos de SchV ocurren de manera esporádica, son unilaterales, y se manifiestan en el quinto decenio de edad. Del total de casos de SchV, los que se presentan en pacientes con NF2 corresponden a entre $2 \%$ y $4 \%{ }^{1}$.

\section{Patogenia}

La causa identificada de los tumores asociados a NF2 son mutaciones del gen que codifica para merlina, ubicado en el brazo largo del cromosoma 22 y entre las bandas q11-13.1. Los schwannomas son causados por alteraciones en las dos copias del gen NF2, aunque en algunos casos en la schwannomatosis aparecen por mutaciones en el gen INI1/SMARCB1. Las mutaciones del gen NF2 también son responsables de $50 \%$ a $60 \%$ de los meningiomas espontáneos, una pequeña proporción de ependimomas espontáneos, y probablemente de todos los schwannomas espontáneos ${ }^{1,10}$.

La merlina (del inglés merlin: Moesin - Ezrin Radixin-Like-proteiN), también Ilamada schwannomina, es una proteína de $69 \mathrm{kDa}$, que muestra una homología a la familia de las proteínas ERM (Ezrin, Radixin, Moesin), las que funcionan como moléculas de anclaje del citoesqueleto a la membrana plasmática ${ }^{1}$. Aunque la merlina se expresa en Ios tejidos afectados por la NF2, también lo hace en tejidos no afectados, como retina, cristalino, testículos, ovario, glándula suprarrenal, y tejido neuronal ${ }^{1}$. La merlina se encuentra primariamente en prolongaciones celulares ricas en actina, y en sitios de contacto célula-célula y célula-matriz extracelular. Se ha especulado que la merlina ejerce 
un rol como proteína supresora de tumores mediante su interacción en los sitios de unión ${ }^{10}$. Diferentes modelos in vitro y con ratones han mostrado como la pérdida de la función de la merlina lleva a tumorogénesis ${ }^{11}$. Se ha demostrado en varias oportunidades que no hay expresión de merlina en tumores causados por mutaciones del gen NF2, y se asume que la ausencia o la inactivación de merlina es fundamental en la génesis tumoral'.

Al comparar células de Schwann humanas normales con células humanas con schwannoma, se ha observado que células deficientes de merlina muestran un patrón de proliferación y de extensión aumentados (el que era reversible a la reintroducción de merlina ${ }^{12}$ ), alteraciones del citoesqueleto, apoptosis disminuida, y adhesión a la matriz extracelular aumentada ${ }^{13}$. Además, la merlina tiene un rol importante en la coordinación de la respuesta de diversos receptores de factores de crecimiento de la familia tirosin kinasa, como el receptor del factor de crecimiento vascular endotelial (VEGFR), la familia de receptores del factor de crecimiento epidérmico (EGFR), factor de crecimiento derivados de plaquetas $B$ (PDGFRß), y el receptor del factor 1 de crecimiento tipo insulina $(\text { IGF1R })^{1,10}$.

\section{Manejo de los pacientes con NF2}

El manejo actual de los SchV comprende desde la observación hasta tratamientos basados en estrategias quirúrgicas. Como los schwannomas son tumores benignos que no responden al tratamiento con quimioterapia clásica, los tratamientos actuales están basados en cirugía o radiocirugía. La cirugía sigue siendo el método de elección para la mayoría de los pacientes esporádicos en términos de terapia citorreductiva, principalmente en lesiones de gran tamaño que provocan efecto de masa o hidrocefalia obstructiva ${ }^{3}$. Los resultados tradicionales presentan, para la primera, pérdida auditiva de hasta $50 \%$ y compromiso del nervio facial de hasta $40 \%$, y para la radiocirugía, tasas de control de hasta $90 \%$, e hipoacusia y compromiso del facial de $5 \%{ }^{14}$.Sin embargo, esto adquiere una dimensión completamente diferente en caso de la NF2, debido a que son tumores que en general se presentan en edades más tempranas, tienden a ser más agresivos, y están asociados a tumores en otras localizaciones, por lo que el tratamiento quirúrgico o radioquirúrgico se debe dejar para situaciones muy seleccionadas. Series de pacientes con NF2 que han sido tratados, muestran resultados peores que series de pacientes con SchV esporádicos, apreciándose preservación auditiva en alrededor de $68 \%$, y control del tumor en alrededor $80 \%$ de los casos ${ }^{1}$. Por esta razón, se considera que el manejo de los pacientes con NF2 debe ser ponderado en cada caso particular, y no existe un tratamiento confiable y seguro. Por ende, se han buscado nuevas estrategias de tratamiento con anticuerpos monoclonales e inhibidores de las cascadas de señalización celular que dependen de merlina.

Los anticuerpos monoclonales son agentes terapéuticos que cada vez adquieren más importancia como terapia oncológica. Poseen variados mecanismos clínicos de acción, dentro de los que se incluye la manipulación de vías de señalización relacionadas con los tumores, propiedades inmunomoduladoras, y activación o inhibición de moléculas del sistema inmune. Por estos mecanismos, los anticuerpos pueden promover la inducción de respuestas inmunes antitumorales ${ }^{15}$.

\section{Avances en el tratamiento}

Se han realizado ensayos clínicos con diferentes tipos de anticuerpos monoclonales en el tratamiento de pacientes con SchV en NF2 (Tabla 2). La estandarización de las respuestas ha sido posible gracias a que en 2008 un grupo de consenso sugirió criterios de respuesta para estudios fase 2 específicos para SchV en pacientes con NF2, que incluía como respuesta imagenológica una reducción de al menos $20 \%$ en resonancia magnética en $\mathrm{T} 1$ con cortes de al menos $3 \mathrm{~mm}$, y como respuesta auditiva se consideraba una mejoría en tests de reconocimientos de listados de 50 palabras $^{16}$.

\section{Bevacizumab (Avastin $\left.{ }^{\circledR}\right)$}

Bevacizumab es un anticuerpo humano IgG1 monoclonal dirigido contra el factor de crecimiento vascular endotelial (VEGF) y que ha sido utilizado como droga antiangiogénica. VEGF es un regulador esencial del crecimiento vascular en condiciones normales y patológicas. En 1993, se demostró in 
Tabla 2. Publicaciones en humanos y animales que investigan el uso de anticuerpos monoclonales para sujetos con neurofibromatosis tipo 2

\begin{tabular}{|c|c|c|c|}
\hline Serie & Año & Anticuerpo & Resultados \\
\hline Clark y cols. & 2008 & $\begin{array}{l}\text { Erlotinib o } \\
\text { Trastuzumab }\end{array}$ & $\begin{array}{l}\text { Ratones tratados por } 12 \text { semanas. Se apreció que } \\
\text { trastuzumab y erlotinib produjeron disminución } \\
\text { crecimiento de SchV. }\end{array}$ \\
\hline Plotkin y cols. & 2009 & Bevacizumab & $\begin{array}{l}10 \text { casos, se apreció disminución volumétrica }(26 \%) \text { en } 6 \\
\text { pacientes, y mejoría auditiva en } 4 \text { casos de } 7 \text { evaluables }\end{array}$ \\
\hline Mautner y cols. & 2010 & Bevacizumab & $\begin{array}{l}2 \text { casos tratados por un año que mostraron reducción } \\
\text { volumétrica (hasta } 43 \% \text { ) que volvió a crecer al suspender } \\
\text { tratamiento }\end{array}$ \\
\hline Wong y cols. & 2010 & $\begin{array}{l}\text { Bevacizumab o } \\
\text { Vandetanib }\end{array}$ & $\begin{array}{l}\text { Líneas celulares humanas y murinas. Se apreció } \\
\text { disminución de diámetro de vasos tumorales, y } \\
\text { aumento de necrosis y apoptosis }\end{array}$ \\
\hline Ammoun y cols. & 2010 & Lapatinib & $\begin{array}{l}\text { Modelo de células humanas de schwannoma in vitro. Se apreció } \\
\text { disminución de la proliferación celular }\end{array}$ \\
\hline Plotkin y cols. & 2010 & Erlotinib & $\begin{array}{l}11 \text { casos, no se apreció disminución volumétrica, pero si } \\
\text { estabilización del crecimiento y deterioro auditivo }\end{array}$ \\
\hline Eminowicz y cols. & 2012 & Bevacizumab & $\begin{array}{l}2 \text { casos tratados previamente, uno con radiocirugía y otro con } \\
\text { otra terapia anti VEGF, ambos con reducción volumétrica de SchV }\end{array}$ \\
\hline
\end{tabular}

SchV: Schwannomas Vestibulares; VEGF: Factor de crecimiento vascular endotelial.

vivo que un anticuerpo monoclonal dirigido contra el VEGF llevó a una supresión del crecimiento tumoral, lo que impulsó la utilización en clínica del bevacizumab. Actualmente está aprobado el uso de esta droga por la FDA como coadyuvante en el tratamiento de cáncer de colon, riñón metastásico y en casos de glioblastomas cerebrales ${ }^{17}$. En una serie de diez pacientes con NF2 y SchV que no eran candidatos a cirugía, se administró bevacizumab endovenoso en dosis de $5 \mathrm{mg} / \mathrm{kg}$ cada dos semanas por 12 meses (rango de 3 a 19 meses). En este estudio, seis pacientes presentaron respuesta imagenológica (definida como reducción de al menos $20 \%$ del volumen del tumor evaluado por resonancia magnética), con una mediana de reducción de volumen de $26 \%$, y cuatro de siete pacientes evaluables presentaron una respuesta en términos de mejoría auditiva ${ }^{18}$. Aunque este estudio ha recibido críticas como la falta de grupo control y no ser un ensayo clínico aleatorizado ${ }^{19,20}$, en términos generales sus resultados pueden ser considerados a lo menos esperanzadores, en la medida que retrasa el crecimiento y el deterioro auditivo. Un reporte de dos pacientes tratados durante tres y seis meses informó una reducción de $40 \%$ del volumen, y mejoría auditiva en ambos casos, sin embargo no se informa si el efecto se mantiene después del tratamiento ${ }^{21}$. En otro estudio con dos pacientes se reporta mantención del volumen tumoral durante un año de tratamiento, pero crecimiento nuevamente al suspender el bevacizumab ${ }^{22}$. En este artículo, en el segundo caso, el paciente desarrolló hipertensión arterial durante el tratamiento, un efecto adverso (toxicidad grado 2) conocido por el uso del bevacizumab ${ }^{23}$. Otro estudio en el que se utilizaron líneas celulares murinas y humanas para evaluar la respuesta a bevacizumab y a vandetanib (un antagonista del receptor de VEGF), informó reducción del diámetro, área y permeabilidad vascular, aumento en la apoptosis y necrosis celular tras dos semanas de tratamiento, y reducción del $50 \%$ de la tasa de crecimiento inicial del tumor ${ }^{24}$. Un reporte de caso en el que se usó bevacizumab por 15 meses para tratar un SchV con respuesta volumétrica pero no audiológica, informó como hallazgo la reducción de un meningioma parietal en $22 \%$, pero no se reporta si la disminución de tamaño se mantiene al suspender el tratamiento ${ }^{25}$. Por último, se ha reportado reducción volumétrica de SchV al utilizar bevacizumab por 12 semanas en un 
caso tratado previamente con radiocirugía y que se ha mantenido estable 8 meses después de finalizado el tratamiento ${ }^{26}$. Además, en este reporte se describe un segundo caso en que se había intentado manejo con PTC299, un inhibidor de la vía del VEGF a nivel posreceptor, con mala respuesta por lo que se le administró un tratamiento de rescate con bevazicumab (5 mg/kg cada 2 semanas), y se describe reducción del volumen tumoral después ocho inyecciones de bevacizumab ${ }^{26}$.

\section{Erlotinib y trastuzumab}

Erlotinib es un inhibidor del EGFR aprobado por la FDA para el tratamiento como coayduvante de cáncer pulmonar de células no pequeñas y cáncer pancreático ${ }^{27}$. En una serie retrospectiva de once pacientes con SchV, no se apreció reducción volumétrica del tumor ni mejoría auditiva, pero sí estabilización del tamaño tumoral27. Utilizando erlotinib y trastuzumab (inhibidor de ErbB2) en un modelo murino, se encontró una reducción del tamaño de los SchV para ambos tratamientos, además de reducción de proliferación celular del SchV al utilizar sólo trastuzumab ${ }^{28}$. Además, se ha descrito el uso de lapatinib (inhibidor dual de EGFR/ ErbB2) en un modelo de células humanas de pacientes con NF2 y SchV esporádicos. En estas células se observó sobreexpresión de la familia de receptores EGFR que fue inhibida por lapatinib ${ }^{29}$. Por último, existe un reporte sobre el efecto de la desregulación de la expresión de varios tipos de microRNAs en células de schwannoma comparadas con tejido nervioso normal, dentro de los cuales aparece el miR-7, el que tendría una función supresora de tumores a través de su relación con la regulación de objetivos de oncogenes, como EGFR y Pak1, por lo que la sobreexpresión de miR-7 inhibiría el crecimiento de células de schwannoma in vitro e in vivo ${ }^{30}$.

\section{CONCLUSIONES}

Para el tratamiento de los SchV en pacientes con NF2 se debe considerar no solamente el tumor en sí, sino el contexto de pacientes extremadamente complejos, con tumores en otras localizaciones, aparición de segundos primarios a futuro y que presentan peores resultados quirúrgicos que los pacientes con SchV esporádicos. Por esta razón, las estrategias de manejo más convencionales (cirugía 0 radiocirugía) deben ser valoradas caso a caso y utilizadas sólo cuando sea necesario, ya que pueden dejar al paciente con morbilidad importante ${ }^{10}$. Los pacientes con NF2 tienen un déficit en la función de merlina, que dado su rol como proteína supresora de tumores en múltiples cascadas intracelulares, se produce una desregulación de la proliferación celular ${ }^{31}$. Por esta razón, aparecen nuevas terapias con anticuerpos monoclonales que regulan las vías de señalización celular de VEGFR mostrando resultados positivos en términos de estabilización del volumen tumoral, e incluso reducción volumétrica y mejoría auditiva. Sin embargo, estos tratamientos aún se encuentran en etapa de investigación, y se desconoce si el efecto reductivo de bevacizumab se mantiene después de suspender el tratamiento. Es probable que en el futuro se utilicen estos tratamientos como terapia coadyuvante para el manejo de la NF2.

\section{BIBLIOGRAFÍA}

1. Hanemann CO. Magic but treatable? Tumours due to loss of Merlin. Brain 2008; 131: 606-15.

2. LALWANI AK. Current Diagnosis and treatment in Otolaryngology - Head and Neck Surgery. Fourth Edition, 2008, McGraw-Hill.

3. Theodosopoulos PV, Pensak ML. Contemporary Management of Acoustic Neuromas. Laryngoscope 2011; 121: 1133-7.

4. Baser ME, Friedman JM, Evans DG. Increasing the specificity of diagnostic criteria for schwannomatosis. Neurology 2006; 66: 730-2.

5. MacCollin M, Chiocca EA, Evans DG, y cols. Diagnostic criteria for schwannomatosis. Neurology 2005; 64: 1838-45.

6. Ho SY, KVETON JF. Acoustic neuroma. Assessment and management. Otolaryngol Clin North Am 2002; 35: 393-404.

7. Zernottı ME, OJeda A, Zernottı M. Schwannomas intrapetrosos del nervio facial. Acta Otorrinolaringol Esp 2005; 56: 434-7.

8. Celedón C, Pardo J, Abarca A, Delano PH. Schwannomas no vestibulares de cabeza y cuello: Presentación de 6 casos clínicos. Rev 
Otorrinolaringol Cir Cabeza Cuello 2011; 71: 4452.

9. Stott C, Albertz N, Aedo C. Neurinoma del acústico (schwanoma vestibular): Revisión y actualización de la literatura. Rev Otorrinolaringol Cir Cabeza Cuello 2008; 68: 301-8.

10. Ammoun S, Hanemann CO. Emerging therapeutic targets in schwannomas and other merlindeficient tumors. Nat Rev Neurol 2011; 7: 3929.

11. Kalamarides M, Nima-Kawakita M, Leblois $\mathrm{H}$, y cols. Nf2 gene inactivation in arachnoidal cells is ratelimiting for meningioma development in the mouse. Genes Dev 2002; 16: 1060-5.

12. Schulze KM, Hanemann CO, Muller HW, Hanenberg $\mathrm{H}$. Transduction of wild-type merlin into human schwannoma cells decreases schwannoma cell growth and induces apoptosis. Hum Mol Genet 2002; 11: 69-76.

13. Flaiz C, Kaempchen K, Matthies C, Hanemann CO. Actin-rich protrusions and non localized GTPase activation in Merlin-deficient schwannomas. J Neuropathol Exp Neurol 2007; 66: 608-16.

14. Combs SE, Volk S, Schulz-Ertner D, Huber PE, Thilmann C, Debus J. Management of acoustic neuromas with fractionated stereotactic radiotherapy (FSRT): long-term results in 106 patients treated in a single institution. Int $J$ Radiat Oncol Biol Phys 2005; 63: 75-81.

15. Weiner LM, Surana R, Wang S. Monoclonal antibodies: versatile eplatforms for cancer immunotherapy. Nature Reviews Immunology 2010; 10: 317-27.

16. Plotkin SR, Halpin C, Blakeley J0, Slattery WH $3^{\text {вр }}$, Welling DB, Chang SM y cols. Suggested response criteriaforphase II antitumor drug studiesfor neurofibromatosis type 2 related vestibular schwannoma. J Neurooncol 2009; 93: 61-77.

17. Ferrara N, Hillan KJ, Gerber HP, Novotny W. Discovery and development of bevacizumab, an anti-VEGF antibody for treating cancer. Nat Rev Drug Discov 2004; 3: 391-400.

18. Plotkin SR, Stemmer-Rachaminov A0, Barker FG $2^{\text {no }}$, Halpin C, Padera TP, Tyrrell A y cols. Hearing improvement after bevacizumab in patients with neurofibromatosis type 2. N Engl J Med 2009; 361: 358-67.
19. Guntinas-Lichius 0 . Hearing improvement after bevacizumab for neurofibromatosis type $2 . \mathrm{N}$ Engl J Med 2009 ;361: 1809-10; authorreply 1810-1.

20. DIAO JS, XIA WS, Guo AZ. Hearing improvement after bevacizumab for neurofibromatosis type 2 . NEngl J Med 2009; 361: 1810; authorreply 18101.

21. Mautner VF, Nguyen R, Kutta H, Fuensterer $C$, BOKEMEYER $C$ y cols. Bevacizumab induces regression of vestibular schwannomas in patients with neurofibromatosis type 2. Neuro Oncol 2010; 12: 14-8.

22. Mautner VF, Nguyen R, Knecht R, Bokemeyer $C$. Radiographic regression of vestibular schwannomas induced by bevacizumab treatment: sustain under continuous drug application and reboundafter drug discontinuation. Ann Oncol 2010; 21: 2294-5.

23. An MM, Zou Z, Shen H, Liu P, Chen ML, CaO YB y coLs. Incidence and risk of significantly raised blood pressure in cancer patients treated with bevacizumab: an updated meta-analysis. Eur $J$ Clin Pharmacol 2010; 66: 813-21.

24. Wong HK, Lahdenranta J, Kamoun WS, Chan AW, McClatchey Al, Plotkin SR y cols. Anti-vascular endothelial growth factor therapies as a novel therapeutic approach to treating neurofibromatosis-related tumors. Cancer Res 2010; 70: 3483-93.

25. Goutagny S, Raymond E, Sterkers 0, Colombani JM, KalAMARIDES M. Radiographic regression of cranial meningioma in a NF2 patient treated by bevacizumab. Ann Oncol 2011; 22: 990-1.

26. Eminowicz GK, Raman R, Conibear J, Plowman PN. Bevacizumab treatment for vestibular schwannomas in neurofibromatosis type two: report of two cases, including responses after prior gamma knife and vascular endothelial growth factor inhibition therapy. J Laryngol Otol 2012; 126: 79-82.

27. Plotkin SR, Halpin C, McKenna MJ, Loeffler JS, Batchelor TT, Barker FG $2^{\mathrm{No}}$. Erlotinib for progressive vestibular schwannoma in neurofibromatosis 2 patients. Otol Neurotol 2010; 31: 1135-43.

28. Clark JJ, Provenzano M, Diggelmenn hr, Xu N, Hansen SS, Hansen MR. The ErbB inhibitors trastuzumab and erlotinib inhibit growth of 
vestibular schwannoma xenografts in nude mice: a preliminary study. Otol Neuroto/2008; 29: 84653.

29. Ammoun S, Cunliffe CH, Allen JC, Chiriboga L, GIancotti FG, ZAGZAG D, y cols. ErbB/HER receptor activation and preclinical efficacy of lapatinib in vestibular schwannoma. Neuro-Oncology 2010; 12: 834-43.
30. Saydam 0, Senol 0, Würdinger T, Mizrak A, Ozdener GB, Stemmer-Rachamimov A0, y cols. miRNA-7 Attenuation in Schwannoma Tumors Stimulates Growth by Upregulating Three Oncogenic Signaling Pathways. Cancer Res 2011; 71: 852-61.

31. Zhou L, Hanemann CO. Merlin, a multi-suppressor from cell membrane to the nucleus. FEBS Lett 2012; 586: 1403-8.

Dirección: Dr. Paul H. Délano

Santos Dumont 999, Servicio de Otorrinolaringología, Hospital Clínico de la Universidad de Chile

E mail: phdelano@gmail.com 